\title{
ONE-SIDED STAR PARTIAL ORDERS FOR BOUNDED LINEAR OPERATORS
}

\author{
JĀNIS CIRULIS
}

Abstract. We compare some recent approaches to transferring the notions of left- and rightstar partial order, introduced for complex matrices in early 90-ies, to bounded linear Hilbert space operators, and discuss a new version of these orders. The main results state that every initial segment of $\mathscr{B}(H)$ under the (new) left-star order is a complete orthomodular sublattice isomorphic to an initial segment of the lattice of closed subspaces of the underlying Hilbert space $H$. We also associate a certain orthogonality relation with the order.

The so called logical order on the set of all self-adjoint operators, introduced by S. Gudder in 2006, turns out to be the restriction of any of both one-sided star orders. Various known results concerning the logical order, in particular, characterizations of the join and meet operations, are extended to the left-star order on $\mathscr{B}(H)$.

Mathematics subject classification (2010): 06A06, 06C15, 46A32, 46C05, 47A05.

Keywords and phrases: bounded linear operator; left-star order; left-star orthogonality; orthogonal projection operator; orthomodular lattice; right-star order; right-star orthogonality.

\section{REFERENCES}

[1] J. Antezana, C. CAno e.a., A note on the star order in Hilbert spaces, Linear Multilinear Algebra 58 (2010), 1037-1051, DOI:10.1080/03081080903227104 .

[2] J.K. BAKSAlary, S.K. Mitra, Left-star and right-star partial ordering, Linear Algebra Appl. 149 (1991), 73-89.

[3] G. Chevalier, Order and orthogonality relations in rings and algebras, Tatra Mountains Math. Publ. 3 (1992), 31-46.

[4] J. CIRULIS, Further remarks on an order of quantum observables, Math. Slovaca (in print; a preprint available as arXiv:1301.0640v2 ).

[5] J. CíRUlis, Lattice operators on Rickart *-rings under the star order, Linear Multilinear Algebra 63 (2015), 497-508, DOI: 10.1080/03081087.2013.873429 .

[6] J. CÍRULIS, On one-side star partial orders on a Rickart *-ring, arXiv:1410.4693v1, 2014.

[7] CH. Deng, Some properties on the star order of bounded operators, J. Math. Anal. Appl. 423 (2014), 32-40, http.//dx.doi.org/10.1016/j.jmaa.2014.09.077 .

[8] Ch. Deng, Sh. WANG, On some characterizations of the partial ordering for bounded operators, Math. Inequal. Appl. 12 (2012), 619-630.

[9] G. Dolinar, A. Guterman, J. Marovt, Monotone transformations on $B(H)$ with respect to the left-star and the right-star partial order, Math. Inequal. Appl. 17 (2014), 573-589.

[10] G. Dolinar, J. Marovt, Star partial order on B(H), Linear Algebra Appl. 434 (2011), 319-326.

[11] S. GudDER, An order for quantum observables, Math. Slovaca 56 (2006), 573-589.

[12] J. Marovt, D.S. Rakić, D.S. Djordjević, Star, left-star, and right-star partial orders in Rickart *-rings, Linear Multilinear Algebra 63 (2015), 343-365, DOI: 10.1080/03081087.2013.866670 .

[13] S.K. Mitra, P. Bhimasanharam, S.B. Malik, Matrix Partial Orders, Shorted Operators and Applications. Singapore: World Scientific, 2010.

[14] S. Pulmannová, E. Vinceková, Remarks on the order for quantum observables, Math. Slovaca 57 (2007), 589-600. 